\title{
Identification of potential molecular targets associated with proliferative diabetic retinopathy
}

Dewang Shao ${ }^{1,2^{*}}$, Shouzhi He ${ }^{1}$, Zi Ye ${ }^{1}$, Xiaoquan Zhu², Wei Sun ${ }^{2}$, Wei Fu ${ }^{2}$, Tianju Ma ${ }^{1}$ and Zhaohui $\mathrm{Li}^{{ }^{*}}$

\begin{abstract}
Background: This study aimed to identify and evaluate potential molecular targets associated with the development of proliferative diabetic retinopathy (DR).
\end{abstract}

Methods: The microarray dataset "GSE60436" generated from fibrovascular membranes (FVMs) associated with proliferative DR was downloaded from the Gene Expression Omnibus database. Differentially expressed genes (DEGs) from the active FVMs and control or inactive FVMs and control were evaluated and co-DEGs were identified using VEEN analysis. Functional enrichment analysis, and protein-protein interactions (PPI) network and module analyses were performed on the upregulated and downregulated coDEGs. Finally, several predictions regarding microRNAs (miRNAs) and transcription factors (TFs) were made to construct a putative TF-miRNA-target network.

Results: A total of 1475 co-DEGs were screened in active/inactive FVM samples, including 461 upregulated and 1014 downregulated genes, which were enriched for angiogenesis [Hypoxia Inducible Factor 1 Subunit Alpha (HIF1A) and Placental Growth Factor (PGF)] and visual perception, respectively. In the case of the upregulated co-DEGs, Kinesin Family Member 11 (KIF11), and BUB1 Mitotic Checkpoint Serine/Threonine Kinase (BUB1) exhibited the highest values in both the PPI network and module analyses, as well as the genes related to mitosis. In the case of downregulated co-DEGs, several G protein subunits, including G Protein Subunit Beta 3 (GNB3), exhibited the highest values in both the PPI network and module analyses. The genes identified in the module analysis were found to be from the signal transduction-related pathways. In addition, we were able to identify four miRNAs and five TFs, including miR-136 and miR-374.

Conclusions: In brief, HIF1A, PGF, KIF11, G protein subunits, and miR-136, miR-374 may all be involved in angiogenesis, retinal endothelial cell proliferation, and visual signal transduction in proliferative DR. This study provides a number of novel insights that may aid the development of future studies dedicated to discovering novel therapeutic targets in proliferative DR.

Keywords: Proliferative diabetic retinopathy, Differentially expressed gene, Functional enrichment analysis, microRNA, Angiogenesis

\footnotetext{
* Correspondence: zhaohuili650@hotmail.com; dewangshao@sina.com ${ }^{1}$ Department of Ophthalmology, The Chinese People's Liberation Army General Hospital, No. 28 Fuxing Road, Haidian District, Beijing 100853, China ${ }^{2}$ Department of Ophthalmology, Air Force Medical Center, PLA, No.15 Chang Yun Gong, Haidian District, Beijing 100089, China
}

(c) The Author(s). 2020 Open Access This article is licensed under a Creative Commons Attribution 4.0 International License, which permits use, sharing, adaptation, distribution and reproduction in any medium or format, as long as you give appropriate credit to the original author(s) and the source, provide a link to the Creative Commons licence, and indicate if changes were made. The images or other third party material in this article are included in the article's Creative Commons licence, unless indicated otherwise in a credit line to the material. If material is not included in the article's Creative Commons licence and your intended use is not permitted by statutory regulation or exceeds the permitted use, you will need to obtain permission directly from the copyright holder. To view a copy of this licence, visit http://creativecommons.org/licenses/by/4.0/ The Creative Commons Public Domain Dedication waiver (http://creativecommons.org/publicdomain/zero/1.0/) applies to the data made available in this article, unless otherwise stated in a credit line to the data. 


\section{Background}

Diabetic retinopathy (DR) is one of the most common microvascular complications in diabetes mellitus, and a primary cause of blindness and visual disturbance globally [1]. DR can be divided into non-proliferative and proliferative forms based on the type of microvascular lesions and related ischemic injury [2]. It has been predicted that the global prevalence of diabetes will continue to grow significantly for the next several decades, as a result of increasing incidence of type 1 diabetes rather than increasing type 2 diabetes which has already reached an epidemic level [3]. The incidence of DR increases with diabetic progression and results from a complex process with various molecular and biochemical participants, including oxidative stress, endoplasmic reticulum stress and mitochondrial damage amongst others [4]. The basic mechanisms underlying DR have been extensively studied, however, effective prevention and therapeutic interventions for this disease are still not available.

At present, DR treatments mainly focus on laser photocoagulation, intravitreal pharmacotherapy, anti-VEGF, and glycemic control [5]. Intravitreal anti-inflammatory agents have been effective in treating DR [6], and intravitreal triamcinolone acetonide shows an antiangiogenic effect toward proliferative DR [7]. Vascular endothelial growth factor (VEGF) plays a critical role in promoting vascular permeability, cell migration as well as proliferation of vascular endothelial cells, vasculogenesis, and angiogenesis [8]. VEGF is intimately involved with the progression of proliferative DR and diabetic macular edema facilitating changes in retinal capillary permeability, and advances in antiVEGF therapies, in age-related macular degeneration, have accelerated the application of anti-VEGF therapies in DR [9]. Oxidative stress is a critical factor in the etiology of DR. Metabolic abnormalities, caused by the increase of glucose concentration in diabetes, can lead to the overproduction of the superoxide radical involved in the uncoupling of mitochondrial electron transport chains, ultimately resulting in oxidative stress $[10,11]$. Antioxidants, including Vitamins and polyphenols, are considered a beneficial therapeutic strategy during the treatment of DR. These compounds inhibit the reactive oxygen species, free radicals and enhance the antioxidant defense system [12]. Despite some progress, DR remains a prevalent vision-threatening disease.

In this study, we used the microarray dataset "GSE60436" generated by Ishikawa $\mathrm{K}$ et al., [13]. In that study, they identified a subset of differentially expressed genes (DEGs) from active fibrovascular membranes (FVMs) and normal retinas or inactive FVMs and normal retinas, these DEGs were then evaluated by functional analysis. In our study, we aim to identify pivotal genes associated with the pathogenesis of proliferative DR and explore their function and upstream and downstream targets. The common DEGs from active/ inactive FVMs and normal retinas were identified, and then subjected to functional enrichment, proteinprotein interaction (PPI) network and module analyses. In addition, the miRNA-target and transcription factors (TFs)target were predicted to explore the potential regulatory relationships.

\section{Methods \\ Study approval}

This study did not use any animal or human participants. All data was generated from public databases.

\section{Data collection and preprocessing}

The gene expression profiling microarray dataset "GSE60436" from FVMs associated with proliferative DR was downloaded from the Gene Expression Omnibus (GEO, http://www.ncbi.nlm.nih.gov/geo/) database. This dataset was made up of a total of nine samples, including three normal human retinas samples (control), three active FVMs samples (age 57.3 \pm 2.1 years; male: female, 1:2; duration of type 2 diabetes, $15.7 \pm 2.1$ years; glycosylated hemoglobin, 8.3\%; vitreous hemorrhage, 1 ; tractional retinal detachment, 2; no anterior chamber neovascularization; no previous anti-VEGF treatment) and three inactive FVM samples (age $48.0 \pm 13$ years; male: female, 1:2; duration of type 2 diabetes, $12.3 \pm 2.5$ years; glycosylated hemoglobin, 6.1\%; tractional retinal detachment; no anterior chamber neovascularization; no previous anti-VEGF treatment), with sequencing data generated using the GPL6884 Illumina HumanWG-6 v3.0 expression beadchip.

Limma package software (Version 3.10.3, http://www. bioconductor.org/packages/2.9/bioc/html/limma.html) was used to process the raw CEL files downloaded from GEO, and data preprocessing was done using the RMA (robust multi-array average) method including background correction, normalization, and expression calculations. The probes were removed when they were not able to be matched to a specific gene symbol, and the average value was taken as the expression value for each gene when different probes matched to the same gene symbol.

\section{DEG screening}

The genes that were differentially expressed in different groups [active-FVMs vs. control (active group); inactiveFVMs vs. control (inactive group)] were analyzed using the empirical Bayes test from the limma package software [14]. The DEGs were screened using a cut-off value of $P<0.05$ and $\mid \log$ fold change (FC) $\mid>2$. The DEGs from both groups were then subjected to VEEN analysis using the VENNY (Version 2.1.0, http://bioinfogp.cnb. csic.es/tools/venny/index.html) online tool. Overlapping genes were considered co-regulated DEGs (co-DEGs) in the following analyses. 


\section{Functional enrichment analysis}

The Database for Annotation, Visualization and Integrated Discovery (DAVID, Version 6.8, https://david-d. ncifcrf.gov/) online tool was used to analyze the KEGG pathway and Gene Ontology annotations for the coDEGs. The number of enriched genes was set as: count $\geq 2$, and $P<0.05$ was considered the threshold value for significantly enriched terms.

\section{PPI network and module analysis}

Search Tool for the Retrieval of Interacting Genes (STRING, Version 11.0, http://www.string-db.org/) was used to predicted the interactions among the co-DEGs with the parameters set to species $=$ human, and PPI score $=0.9$ (highest confidence). The PPI network was constructed using Cytoscape software (version 3.2.0, http://www.cytoscape.org/) based on the interactions retrieved from STRING. In addition, the MCODE plugin [15] (Version 1.4.2, http:// apps.cytoscape.org/apps/MCODE) from Cytoscape was used to identify the significant modules, with scores $>10$, and was followed by functional enrichment analysis.

\section{TF-miRNA-target network construction}

The overrepresentation enrichment analysis method from WebGestalt [16] (http://www.webgestalt.org/) was used to predict the TF- and miRNA-target interactions for all of the genes from the significant modules using a cut-off value of $P<0.05$. Then, the TF-miRNA-Target regulatory network was constructed by combining miRNA-target and TF-target regulatory interactions.

\section{Results \\ Data preprocessing and DEG screening}

The expression values for all the genes from the nine samples were normalized using the RMA method, and values with an unchanged position in the boxplot were used for subsequent analysis, as this can be used as a proxy for normalization (Fig. 1a). A total of 2025 DEGs were identified in the active group, including 758 upregulated DEGs and 1297 downregulated DEGs. Similarly, 1961 DEGs (757 upregulated and 1204 downregulated) were identified in the inactive group. Fig. 1b shows the heat maps of those DEGs, and reveals that the DEGs can be easily distinguished from each of the samples. In addition, the VEEN analysis, which was performed to identify common DEGs in the two groups, identified 1475 overlapping or coDEGs. Of these 461 genes were upregulated, and 1014 genes were downregulated (Fig. 1c).

\section{Functional enrichment analysis}

The upregulated co-DEGs were significantly enriched for 148 GO-biological processes (GO-BP) and 41 KEGG pathways. Including "GO:0030198 extracellular matrix organization", "GO:0001525 angiogenesis" and pathways "hsa04512: ECM-receptor interaction", "hsa04151: PI3KAkt signaling pathway". In addition, the downregulated co-DEGs were significantly enriched for 150 GO-BP

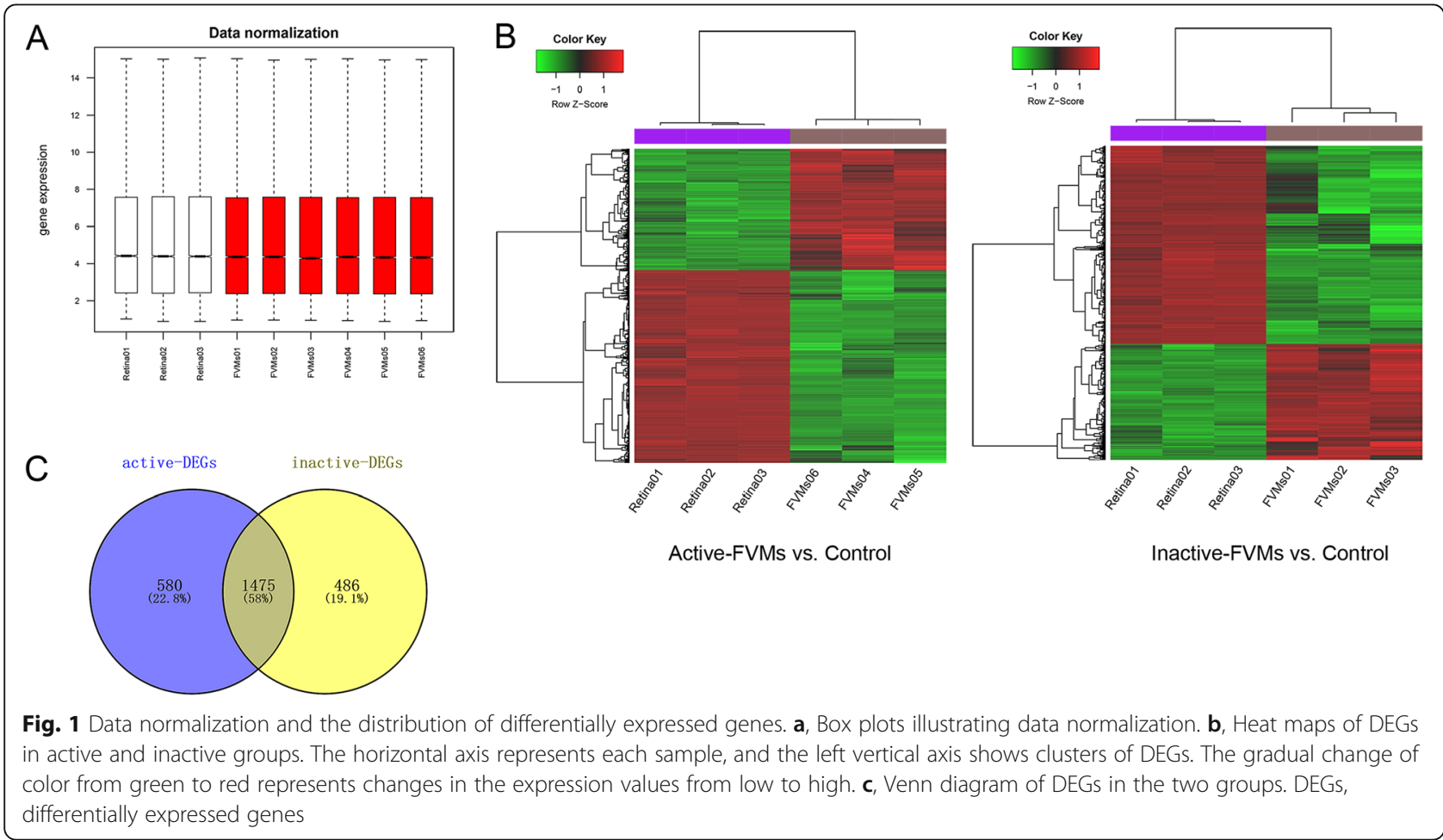


terms and 40 KEGG pathways including, "GO: 0007601 visual perception", "GO:0001523 retinoid metabolic process" and pathways "hsa04744: Phototransduction" and "hsa04724: Glutamatergic synapse". Table 1 summarizes the top $10 \mathrm{GO}-\mathrm{BP}$ terms and KEGG pathways.

\section{PPI network and module analysis}

For the upregulated co-DEGs.

The upregulated co-DEGs were used to retrieve various predicted interactions, which were then used to create the PPI network. This network consisted of 202 nodes and 751 interactions (Supplemental Fig. 1). Moreover, two significant modules with score $\geq 10$ were identified. ModuleA (score $=18.4$ ) contained 21 nodes and 184 interactions, and module- $\mathrm{B}($ score $=12)$ included 12 nodes and 66 interactions (Fig. 2). Kinesin Family Member 11 (KIF11), BUB1 Mitotic Checkpoint Serine/Threonine Kinase (BUB1) and Cyclin B2 (CCNB2) were the hub genes with the highest degree of relevance in both the PPI network and module-A. The genes in module-A were found to be related to three KEGG pathways and 25 GO-BP terms, including "GO:0007067 mitotic nuclear division" and "GO: 0051301 cell division". Additionally, the genes in module$B$ were found to participated in three KEGG pathways and eight GO-BP terms, including "GO:0030198 extracellular matrix organization".

For the downregulated co-DEGs.

The PPI network constructed using downregulated coDEGs contained 349 nodes and 870 interactions (Supplemental Fig. 2), from which two significant modules with score $\geq 10$ were also identified. Module-A (score $=18.24$ ) contained 26 nodes and 228 interactions and module-B $($ score $=10)$ included 10 nodes and 45 interactions (Fig. 3). $\mathrm{G}$ protein subunits were the hub genes with the highest degree of conservation in both the PPI network and moduleA, including G Protein Subunit Beta 3 (GNB3), GNB5, G Protein Subunit Gamma 13 (GNG13), GNG8, GNG17, and GNG3. The genes in module-A were found to be significant in 16 KEGG pathways and 34 GO-BP terms, including "hsa04725: Cholinergic synapse" and "GO:0007186 G -protein coupled receptor signaling pathway". Similarly, the genes in module- $\mathrm{B}$ were found to be associated with two KEGG pathways and nine GO-BP terms. Fig. 4 shows the enriched KEGG pathways and top five GO-BP terms for the genes found in the significant modules.

\section{TF-miRNA-target network construction}

The genes from the significant modules were used to predicted the TF- and miRNA-target interactions, which were then used to construct the TF-miRNA-target network (Fig. 5). The TF-miRNA-target network consisted of 33 nodes and 27 interactions, from which we were able to identify four miRNAs and five TFs, which may interact with up to 24 novel genes. GNG3 was shown to be regulated by miR-136, and Growth Factor Independent 1 Transcriptional Repressor (GFI1). KIF20A was regulated by miR-374, and so on.

\section{Discussion}

In this study, we identified 1475 co-DEGs from active/inactive-FVMs samples. The upregulated co-DEGs were found to participate in angiogenesis [Hypoxia Inducible Factor 1 Subunit Alpha (HIF1A) and Placental Growth Factor (PGF)] and mitosis-related processes (the genes in upregulated module-A, including KIF11 and BUB1). The downregulated co-DEGs were enriched in $\mathrm{G}$-protein coupled receptor signaling and other pathways (The genes in downregulated module-A, including GNB3 and GNG8).

The important pathological features of proliferative DR including abnormal growth of retinal blood vessels and angiogenesis have all been linked to VEGF signaling. High blood sugar can trigger hypoxia in retinal tissues, and this hypoxia acts as a crucial factor in the regulation of VEGF-induced angiogenesis through the production of HIF [17]. HIF1A is an hypoxia induced TF and the lack of HIF1A suppresses the formation of the retinal intermediate vascular plexus in mouse models [18]. PGF, a homologue of VEGF, plays an important role in placental development, and has been shown to be involved in pathological angiogenesis both in ocular and nonocular cancers [19]. In addition, increased expression of PGF has also been observed in the vitreous of DR patients [19]. Bender et al., revealed that PGF and VEGFA are both essential for follicular angiogenesis in primates, and neither can function alone and result in normal follicular angiogenesis [20]. Huang et al. showed that PGF negatively effects retinal endothelial cell barrier function by activating VEGFR1 and VEGFR2 and inhibiting glucose-6-phosphate dehydrogenase and the antioxidant pathways [21]. Ishikawa $\mathrm{K}$ et al., reported that the genes that were significantly upregulated in active FVMs were predominantly part of the angiogenesis process [13]. Therefore, we speculate that PGF and VEGF are important in the progression of FVM to proliferative DR.

MicroRNAs (miRNAs) are small non-coding RNAs of around 22 nucleotides (nt) in length, which mediate the expression of protein-coding genes, and are becoming increasingly popular biomarkers used in both prognostic and diagnostic assays $[22,23]$. Targets for miR-136 and miR374 were significantly enriched in the co-DEGs identified in this study. However, the relationship between miR-136 or miR-374 and DR is not well reported, and this is the first publication linking them. miR-136 is highly expressed in pre-eclampsia and inhibits the formation of HUVEC capillaries via dysregulation of VEGF. In addition, the expression levels of miR-136 inversely correlates with VEGF [24]. In paclitaxel-resistant ovarian cancer cells, pre-miR-136 transfection significantly decreased angiogenesis and induced 
Table 1 The top 10 significantly enriched KEGG pathways and GO-BP terms of co-DEGs

\section{(a) Up-regulated co-DEGs}

Category Terms

GO_BP

GO_BP

GO_BP

GO_BP

GO_BP

GO_BP

GO_BP

GO_BP

GO_BP

GO_BP

KEGG_pathway

KEGG_pathway

KEGG_pathway

KEGG_pathway

KEGG_pathway

KEGG_pathway

KEGG_pathway

KEGG_pathway

KEGG_pathway

KEGG_pathway
GO:0030198 extracellular matrix organization

GO:0006954 inflammatory response

GO:0007155 cell adhesion

GO:0006955 immune response

GO:0001525 angiogenesis

GO:0030574 collagen catabolic process

GO:0030199 collagen fibril organization

GO:0071347 cellular response to interleukin-1

GO:0045766 positive regulation of angiogenesis

GO:0050900 leukocyte migration

hsa04512:ECM-receptor interaction

hsa04510:Focal adhesion

hsa04151:PI3K-Akt signaling pathway

hsa04060:Cytokine-cytokine receptor interaction

hsa05146:Amoebiasis

hsa04620:Toll-like receptor signaling pathway

hsa05222:Small cell lung cancer

hsa05162:Measles

hsa04640:Hematopoietic cell lineage

hsa05205:Proteoglycans in cancer

(b) Down-regulated co-DEGs

GO_BP
GO_BP
GO_BP
GO_BP
GO_BP
GO_BP
GO_BP
GO_BP
GO_BP

GO_BP

KEGG_pathway

KEGG_pathway

KEGG_pathway

KEGG_pathway

KEGG_pathway

KEGG_pathway

KEGG_pathway

KEGG_pathway

KEGG_pathway

KEGG_pathway
GO:0007601 visual perception

GO:0001523 retinoid metabolic process

GO:0006810 transport

GO:0007602 phototransduction

GO:0042462 eye photoreceptor cell development

GO:0050896 response to stimulus

GO:0070588 calcium ion transmembrane transport

GO:0034220 ion transmembrane transport

GO:0022400 regulation of rhodopsin mediated signaling pathway

GO:0042572 retinol metabolic process

hsa04744:Phototransduction

hsa04724:Glutamatergic synapse

hsa04727:GABAergic synapse

hsa00350:Tyrosine metabolism

hsa04723:Retrograde endocannabinoid signaling

hsa04721:Synaptic vesicle cycle

hsa04261:Adrenergic signaling in cardiomyocytes

hsa05033:Nicotine addiction

hsa04020:Calcium signaling pathway

hsa04725:Cholinergic synapse

\begin{tabular}{|c|c|}
\hline$P$ value & Genes \\
\hline 1.10E-19 & PXDN, TNF, LUM, TNC, COL3A1... \\
\hline $9.11 \mathrm{E}-14$ & C3AR1, CCL3, TNF, NMI, TLR1... \\
\hline 4.44E-12 & TNC, ITGA11, POSTN, IL32, CXCR3... \\
\hline 3.50E-09 & PXDN, CCL3, TNF, TLR1, CXCL9... \\
\hline $1.37 \mathrm{E}-08$ & PIK3CG, COL4A2,PGF, HIF1A, PIK3CA... \\
\hline 3.59E-08 & COL4A2, COL4A1, MMP9, COL3A1, COL6A3... \\
\hline 4.34E-08 & LUM, COL3A1, COL1A2, COL1A1, LOX... \\
\hline $1.04 \mathrm{E}-06$ & ADAMTS7, CCL3, HIF1A, PTGIS, CCL3L1... \\
\hline $2.34 \mathrm{E}-06$ & C3AR1, CYBB, HIF1A, PTGIS, PGF... \\
\hline 4.37E-06 & C3AR1, OLR1, MMP9, F2RL1, ITGA4... \\
\hline $6.58 \mathrm{E}-17$ & COL4A2, COL4A1, COL3A1, ITGA11, ITGA1... \\
\hline 1.43E-12 & PGF, TNC, COL3A1, ITGA11, PIK3CA... \\
\hline 3.94E-11 & PGF, OSMR, PIK3CA, COL3A1, ITGA11... \\
\hline $1.04 \mathrm{E}-08$ & CCL3, TNF, TNFSF4, TNFRSF12A, OSMR... \\
\hline $5.63 \mathrm{E}-08$ & PIK3CG, COL4A2, TNF, COL4A1, COL3A1... \\
\hline 4.16E-07 & PIK3CG, IKBKE, CCL3, TNF, CCL3L1... \\
\hline $1.96 \mathrm{E}-06$ & PIK3CG, E2F2, COL4A2, LAMA4, COL4A1... \\
\hline 5.63E-06 & PIK3CG, CD3G, FASLG, CDK6, TLR7... \\
\hline $1.71 \mathrm{E}-05$ & TNF, CD3G, CD36, ITGA5, FCGR1A... \\
\hline 2.71E-05 & PIK3CG, TNF, LUM, MMP9, ITGA2... \\
\hline $5.92 \mathrm{E}-40$ & SLC45A2, PITPNA, MYO7A, RRH, RP1L1... \\
\hline $6.62 \mathrm{E}-11$ & RBP4, OPN1LW, RBP1, RBP3, OPN1MW... \\
\hline 7.57E-11 & RBP7, CRABP1, GABRB3, GRIK1, RBP1... \\
\hline 4.43E-10 & GUCA1B, UNC119, GUCA1A, TRPC3, OPN1LW... \\
\hline 7.94E-10 & GNAT1, TULP1, CRB1, FSCN2, MYO7A... \\
\hline 8.03E-10 & SLC45A2, CLDN19, IRX5, SLC24A5, RPGRIP1... \\
\hline 4.64E-08 & TRPM3, SLC8A2, TRPC3, CACNG7, SLC24A5 .. \\
\hline 1.57E-07 & FXYD3, ATP1B1, GRIK1, GABRB3, ATP1B2... \\
\hline 2.34E-07 & GUCA1B, GNAT1, PDE6A, GUCA1A, PDE6B... \\
\hline 3.37E-07 & $\mathrm{RDH} 12, \mathrm{RDH} 8, \mathrm{ALDH} 1 \mathrm{~A} 2, \mathrm{TTR}, \mathrm{RBP} 4 \ldots$ \\
\hline 3.68E-10 & GNAT1, GUCA1B, GUCA1A, GUCA1C, CNGB1... \\
\hline $7.66 \mathrm{E}-08$ & SLC38A3, GNAO1, GRIK1, ADCY5, GNG13... \\
\hline 1.03E-07 & GABRG2, GABARAPL1, SLC38A3, GNAO1, GABRB3... \\
\hline $1.48 \mathrm{E}-07$ & DCT, DDC, TYRP1, TYR, PNMT... \\
\hline 1.36E-06 & GABRG2, GNAO1, GABRB3, ADCY5, GABRB1... \\
\hline 1.37E-05 & CPLX4, SLC17A7, SLC32A1, ATP6V1C2, SLC17A8... \\
\hline 2.72E-05 & ATP1B1, ATP1B2, MYL3, TNNC1, ADCY5... \\
\hline 4.19E-05 & SLC17A7, SLC32A1, SLC17A8, GABRG2, GABRR1... \\
\hline 9.36E-05 & PTGER1, SLC8A2, SLC25A4, CCKBR, TNNC1... \\
\hline 3.05E-04 & GNAO1, ADCY5, GNG13, KCNJ14, GNG8... \\
\hline
\end{tabular}




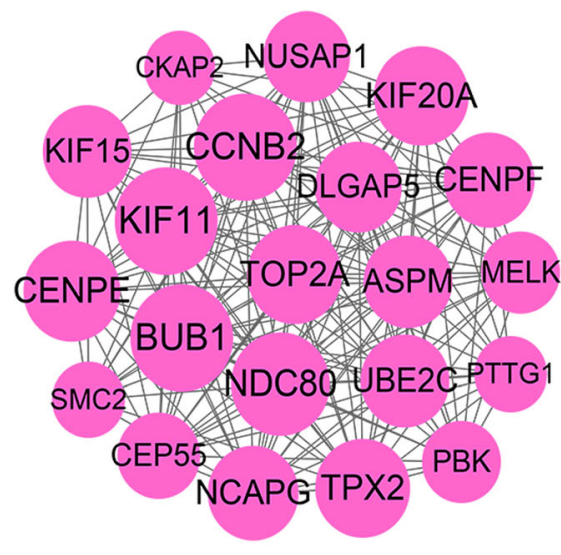

Module A

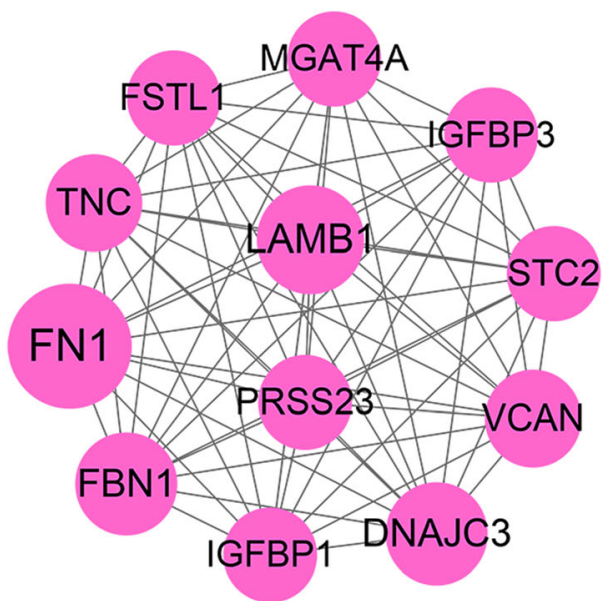

Module B

Fig. 2 The significant modules identified in the PPI network of upregulated co-DEGs. Node size represents the degree score; lines represent interactions; co-DEGs, co-regulated differentially expressed genes

apoptosis, and regulation of miR-136 expression could resensitize paclitaxel-resistant cells [25]. C-C Motif Chemokine Ligand 3 represses the expression of miR-374b, which subsequently accelerates VEGF-A level and angiogenesis in osteosarcoma cells [26]. Thus, we speculate that miR-136 and miR-374 may participate in the regulation of angiogenesis in proliferative DR.

KIF11 is a member of the kinesin-like protein family. Mutations in KIF11 have been shown to cause familial exudative vitreoretinopathy associated with retinal detachment [27]. Birtel et al., revealed that KIF11 is important in both ocular development and the maintenance of retinal morphology and function and defects in this protein have been associated with retinal ciliopathy [28]. KIFs are motor proteins involved in the activities of the centrosome and spindle during mitosis. In our study, KIFs (KIF11, KIF15, KIF20A) and other mitosis-associated genes, like BUB1, were significantly enriched in one module involved in cell division and mitotic nuclear division. The proliferation of retinal endothelial cells is a significant event in the progression of DR [29]. In addition, a previous study has shown that high glucose significantly increases the proliferation of retinal endothelial cells and the expression levels of VEGF, also, it was more effective under intermittent high glucose, than constant high glucose conditions [30]. Therefore, we suggest that the genes in this module play a key role in proliferative DR through the regulation of the proliferation of retinal endothelial cells.

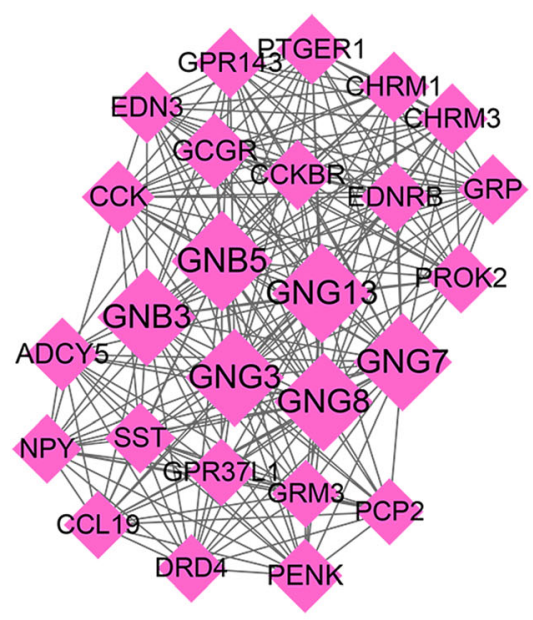

Module A

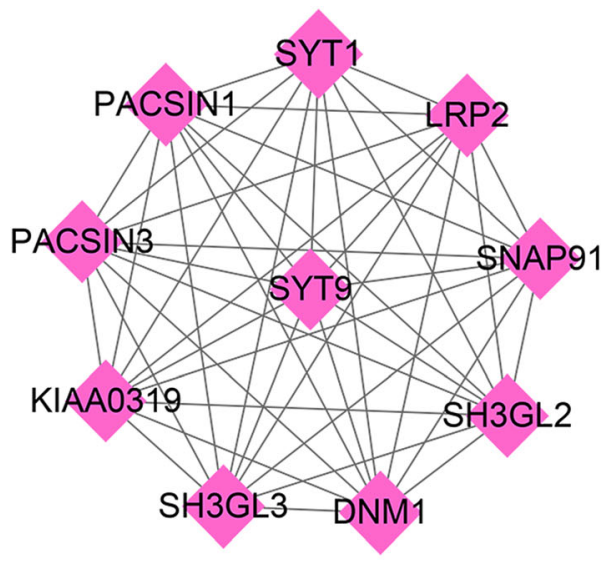

Module B

Fig. 3 The significant modules identified in the PPI network of downregulated co-DEGs. Node size represents the degree score; lines represent interactions. co-DEGs, co-regulated differentially expressed genes 

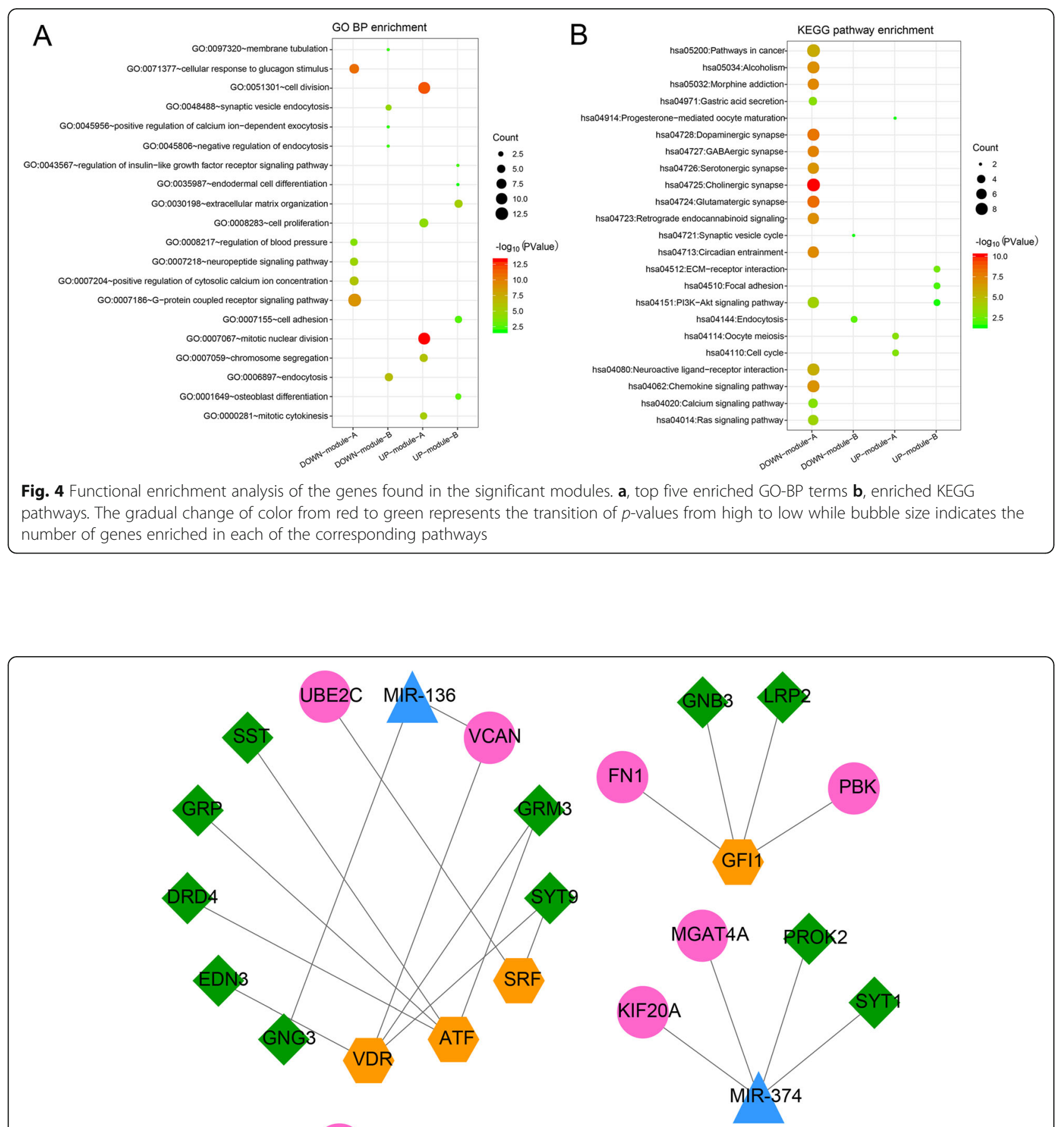

FBN1

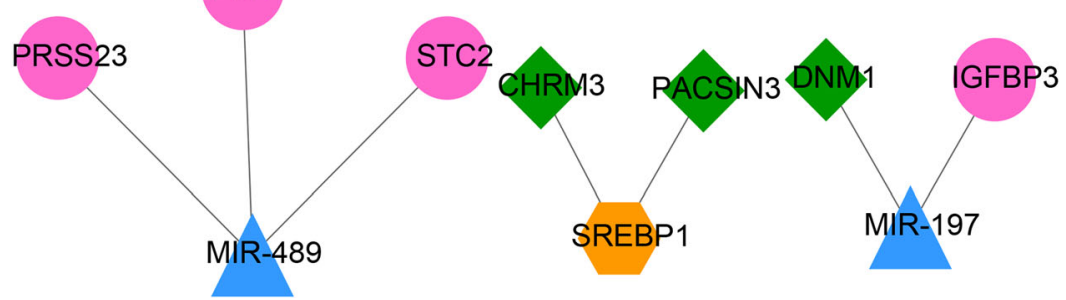

Fig. 5 TF-miRNA-Target regulatory network. Blue triangles represent the miRNA; orange hexagons represent TFs; pink circles represent the upregulated genes and green rhombi represent the downregulated genes 
$\mathrm{G}$ proteins are a kind of heterotrimeric guanine nucleotide-binding protein consisting of three subunits (alpha, beta and gamma subunits), which play an important role in intracellular signal transduction, including insulin, epinephrine, dopamine signaling molecule transduction [31]. GNB3 encodes G Protein Subunit Beta 3. The GNB3 gene polymorphisms, including C825T, have been linked to type 2 diabetes mellitus and its complications [32]. GNB3 knockout lead to the malfunction of cone photoreceptors and ON-bipolar cells in murine retinas [33]. In addition, it was revealed that a naturally occurring mutation in GNB3 results in retinal degeneration in chickens [34]. A homozygous missense variant in GNB3 results in a unique stationary retinal disorder with dual anomalies in visual processing, night blindness and photophobia [35]. G protein subunits (GNB3, GNB5, GNG3, GNG7, GNG8, GNG13) are downregulated in proliferative $\mathrm{DR}$, and significantly enriched in module A which is associated with cellular responses to glucagon stimuli including $\mathrm{G}$ - protein coupled receptor signaling pathways and other biological processes. Therefore, we conclude that $\mathrm{G}$ proteins may play a crucial role in visual signal transduction in proliferative DR.

Although this study has made several novel observations, all the results were based on bioinformatics analysis, and further experimental verification is required. In addition, in an ideal situation, healthy retinas, nonproliferative diabetic retinas and autologous retinas should be used as controls for a more comprehensive comparison with retinas from subjects with FVM-associated proliferative DR. However, such microarray studies are limited by challenges in obtaining samples. Additionally, the small sample size in this study may also limit the application of our observations. Genes that are important for proliferative DR might be excluded as a result of the small sample size and inter-sample variation.

\section{Conclusions}

In brief, we investigated differentially expressed gene profiles in proliferative DR. Several genes, including HIF1A, PGF, KIF11, G protein subunits, and miR-136, miR-374 were found to be involved in angiogenesis, retinal endothelial cell proliferation, and visual signal transduction in proliferative DR. These findings could provide valuable insight and foundations for the study of proliferative DR's pathomechanism and may provide an idea of potential therapeutic targets for its treatment.

\section{Supplementary information}

Supplementary information accompanies this paper at https://doi.org/10 1186/s12886-020-01381-5.

Additional file 1 Figure S1 The PPI network of upregulated co-DEGs. Node size represents the degree score; lines represent interactions; coDEGs, co-regulated differentially expressed genes.
Additional file 2 Figure S2. The PPI network of downregulated coDEGs. Node size represents the degree score; lines represent interactions; co-DEGs, co-regulated differentially expressed genes.

\begin{abstract}
Abbreviations
DR: Diabetic retinopathy; FVMs: Fibrovascular membranes; DEGs: Differentially expressed genes; PPI: Protein-protein interactions; miRNAs: Micrornas;

TFs: Transcription factors; HIF1A: Hypoxia Inducible factor 1 subunit alpha; PGF: Placental growth factor; KIF11: Kinesin family member 11; BUB1: BUB1 mitotic checkpoint serine/threonine kinase; GNB3: G Protein Subunit Beta 3; VEGF: Vascular endothelial growth factors; co-DEGs: Co-regulated DEGs; GOBP: GO-biological processes; KIF11: Kinesin family member 11; CCNB2: Cyclin B2; GNG13: G protein subunit gamma 13; GFI1: Growth factor independent 1 transcriptional repressor
\end{abstract}

\section{Acknowledgements}

Not applicable.

\section{Authors' contributions}

Conceptualization and manuscript preparation were completed by DWS XQZ and ZHL. Acquisition of data was completed by ZY. Analysis and interpretation of data was completed by DWS. Statistical analysis was done by WS and TJM. Revision of manuscript for important intellectual content was undertaken by SZH and WF. All authors have read and approved the manuscript in its current state.

\section{Funding}

This work was supported by the National Natural Science Foundation of China (No. 81870640) and the Beijing Natural Science Foundation (No. 7182152). The funders play the roles of collection, analysis, and interpretation of data.

\section{Availability of data and materials}

The dataset "GSE60436" were downloaded from GEO data NCBI Gene Expression Omnibus repository. All the data of the current study are available at https://pan. baidu.com/s/12j9WMgvoBapXW3WGCSGfhQ, and the extraction code can be obtained from the corresponding author upon reasonable request.

\section{Ethics approval and consent to participate}

This study was approved by the Ethics Committee from The Chinese People's Liberation Army General Hospital and Air Force Medical Center.

Consent for publication

Not applicable.

Competing interests

The authors have no conflicts of interest to declare.

Received: 17 November 2019 Accepted: 10 March 2020

Published online: 14 April 2020

\section{References}

1. Laud K, Shabto U, Tello C: Diabetic Retinopathy. In: Principles of Diabetes Mellitus. edn. Edited by Poretsky L. Cham: Springer International Publishing; 2016:1-18.

2. Stitt AW, Curtis TM, Chen M, Medina RJ, Mckay GJ, Jenkins A, Gardiner TA, Lyons TJ, Hammes HP, Simó R. The progress in understanding and treatment of diabetic retinopathy. Prog Retin Eye Res. 2016;51:156-86.

3. Duh EJ, Sun JK, Stitt AW. Diabetic retinopathy: current understanding, mechanisms, and treatment strategies. Jci Insight. 2017;2(14):e93751.

4. Petrovski G, Kaarniranta K, Petrovič D. Oxidative stress, epigenetics, environment, and epidemiology of diabetic retinopathy. Journal of Diabetes Research. 2017;2017: 6419357.

5. Stewart MW. Treatment of diabetic retinopathy: recent advances and unresolved challenges. World J Diabetes. 2016;7(16):333-41.

6. Kumar B, Gupta SK, Saxena R, Srivastava S. Current trends in the pharmacotherapy of diabetic retinopathy. J Postgrad Med. 2016;58(2):132-9.

7. Jonas JB. Intravitreal triamcinolone acetonide for diabetic retinopathy. Dev Ophthalmol. 2007;39:96-110.

8. Gupta N, Mansoor S, Sharma A, Sapkal A, Sheth J, Falatoonzadeh P, Kuppermann B, Kenney M. Diabetic retinopathy and VEGF. Open Ophthalmol J. 2013;7(1):4-10. 
9. Nicholson BP, Schachat AP. A review of clinical trials of anti-VEGF agents for diabetic retinopathy. Graefes Arch Clin Exp Ophthalmol. 2010;248(7):915-30.

10. Calderon GD, Juarez OH, Hernandez GE, Punzo SM, ZDDL C. Oxidative stress and diabetic retinopathy: development and treatment. Eye. 2017;31(8):1122-30.

11. Guzman DC, Olguín HJ, García EH, Peraza AV, Dz DLC, Soto MP. Mechanisms involved in the development of diabetic retinopathy induced by oxidative stress. Redox Rep. 2017;22(1):10-6.

12. Li C, Miao X, Li F, Wang S, Liu Q, Wang Y, Sun J. Oxidative Stress-Related Mechanisms and Antioxidant Therapy in Diabetic Retinopathy. Oxid Med Cell Longev. 2017;(11):9702820.

13. Keijiro I, Shigeo Y, Yoshiyuki K, Yedi Z, Takahito N, Shintaro N, Yukio S, Yuji $\mathrm{O}$, Hiroaki N, Koichi A. Microarray analysis of gene expression in fibrovascular membranes excised from patients with proliferative diabetic retinopathy. Invest Ophthalmol Vis Sci. 2015;56(2):932.

14. Smyth G.K. (2005) limma: Linear Models for Microarray Data. In: Gentleman R, Carey VJ, Huber W, Irizarry RA, Dudoit S. (eds) Bioinformatics and Computational Biology Solutions Using R and Bioconductor. Statistics for Biology and Health. Springer: New York.

15. Bandettini WP, Kellman P, Mancini C, Booker OJ, Vasu S, Leung SW, Wilson JR, Shanbhag SM, Chen MY, Arai AE. MultiContrast delayed enhancement (MCODE) improves detection of subendocardial myocardial infarction by late gadolinium enhancement cardiovascular magnetic resonance: a clinical validation study. J Cardiovasc Magn Reson Official J Soc Cardiovasc Magn Reson. 2012;14(1):83.

16. Nucleic Acids Res. 2005, 33(Web Server issue):W741-8.

17. Crawford TN, Rd AD, Kerrison JB, Jablon EP. Diabetic retinopathy and angiogenesis. Curr Diabetes Rev. 2009;5(1):8-13.

18. Christian C, Markus T, Christina L, Sandrine J, Marijana S, Christian G. HIF1A is essential for the development of the intermediate plexus of the retinal vasculature. Invest Ophthalmol Vis Sci. 2011;52(5):2109-17.

19. Nguyen QD, De FS, Behar-Cohen F, Lam WC, Li X, Reichhart N, Ricci F, Pluim J, Li WW. Placental growth factor and its potential role in diabetic retinopathy and other ocular neovascular diseases. Acta Ophthalmol. 2018:96(1):e1-9.

20. Bender HR, Trau HA, Duffy DM. Placental growth factor is required for ovulation, Luteinization, and angiogenesis in primate ovulatory follicles. Endocrinology. 2018;159(2):710-22.

21. Huang H, Lennikov A, Saddala MS, Gozal D, Grab DJ, Khalyfa A, Fan L. Placental growth factor negatively regulates retinal endothelial cell barrier function through suppression of glucose-6-phosphate dehydrogenase and antioxidant defense systems. FASEB J. 2019;33(12):13695-709.

22. Joglekar MV, Januszewski AS, Jenkins AJ, Hardikar AA. Circulating microRNA biomarkers of diabetic retinopathy. Diabetes. 2016;65(1):22-4.

23. Farr RJ, Januszewski AS, Joglekar MV, Liang H, McAulley AK, Hewitt AW, Thomas HE, Loudovaris T, Kay TWH, Jenkins A, et al. A comparative analysis of high-throughput platforms for validation of a circulating microRNA signature in diabetic retinopathy. Sci Rep. 2015;5:10375.

24. Ji L, Zhang L, Li Y, Guo L, Cao N, Bai Z, Song Y, Xu Z, Zhang J, Liu C. MiR136 contributes to pre-eclampsia through its effects on apoptosis and angiogenesis of mesenchymal stem cells. Placenta. 2017:50:102-9.

25. An H, Jeong J, Lee $M$, Song J, Jung $Y$, Kim Y, Kwon A, Huh J, Kim K, Kang H. 275: MiR-136 targeting Notch3 is involved in chemoresistance and angiogenesis in ovarian cancer cells. Eur J Cancer. 2014;50(1):S65.

26. Liao Y-Y, Tsai H-C, Chou P-Y, Wang S-W, Chen H-T, Lin Y-M, Chiang IP, Chang T-M, Hsu S-K, Chou M-C, et al. CCL3 promotes angiogenesis by dysregulation of miR-374b/ VEGF-A axis in human osteosarcoma cells. Oncotarget. 2015;7(4):4310-25.

27. Hu H, Xiao X, Li S, Jia X, Guo X, Zhang Q. KIF11 mutations are a common cause of autosomal dominant familial exudative vitreoretinopathy. $\mathrm{Br} J$ Ophthalmol. 2016;100(2):278-83.

28. Birtel J, Gliem M, Mangold E, Tebbe L, Spier I, Müller PL, Holz FG, Neuhaus C, Wolfrum U, Bolz HJ. Novel insights into the Phenotypical Spectrum of KIF11-associated retinopathy, including a new form of retinal Ciliopathy. Invest Ophthalmol Vis Sci. 2017;58(10):3950.

29. West DC, Kumar S. Endothelial cell proliferation and diabetic retinopathy. Lancet. 1988;331(8587):715-6.

30. Sun J, Xu Y, Sun S, Sun $Y$, Wang $X$. Intermittent high glucose enhances cell proliferation and VEGF expression in retinal endothelial cells: the role of mitochondrial reactive oxygen species. Mol Cell Biochem. 2010; 343(1-2):27-35.

31. Gilman AG. G proteins: transducers of receptor-generated signals. Annu Rev Biochem. 1986;56(56):615-49.
32. Rizvi S, Raza ST, Rahman Q, Mahdi F. Role of GNB3, NET, KCNJ11 , TCF7L2 and GRL genes single nucleotide polymorphism in the risk prediction of type 2 diabetes mellitus. Biotech. 2016;6(2):255.

33. Arno G, Holder GE, Chakarova C, Kohl S, Pontikos N, Fiorentino A, Plagnol V, Cheetham ME, Hardcastle AJ, Webster AR. Recessive retinopathy consequent on mutant G-protein $\beta$ subunit 3 (GNB3). Jama Ophthalmol. 2016;134(8):924

34. Hemanth T, Manir A, Paul G, Hocking PM, Burt DW, Inglehearn CF, Lester $\mathrm{DH}$. Mutation in the guanine nucleotide-binding protein beta-3 causes retinal degeneration and embryonic mortality in chickens. Invest Ophthalmol Vis Sci. 2006:47(11):4714.

35. Banin E, Bocquet B, Debaere E, Casteels I, Defoort-Dhellemmes S, Drumare I, Friedburg C, Gottlob I, Jacobson S, Kellner U. Biallelic mutations in GNB3 cause a unique form of autosomal-recessive congenital stationary night blindness. Am J Hum Genet. 2016;98(5):1011-9.

\section{Publisher's Note}

Springer Nature remains neutral with regard to jurisdictional claims in published maps and institutional affiliations.

Ready to submit your research? Choose BMC and benefit from:

- fast, convenient online submission

- thorough peer review by experienced researchers in your field

- rapid publication on acceptance

- support for research data, including large and complex data types

- gold Open Access which fosters wider collaboration and increased citations

- maximum visibility for your research: over $100 \mathrm{M}$ website views per year

At $\mathrm{BMC}$, research is always in progress.

Learn more biomedcentral.com/submissions 